\title{
Histoire du Coran. Texte et transmission
}

\section{François Déroche}

\section{OpenEdition}

\section{Journals}

Édition électronique

URL : https://journals.openedition.org/annuaire-cdf/15893

DOI : $10.4000 /$ annuaire-cdf.15893

ISBN : 978-2-7226-0572-5

ISSN : 2109-9227

Éditeur

Collège de France

Édition imprimée

Date de publication : 30 décembre 2020

Pagination : 293-309

ISBN : 978-2-7226-0516-9

ISSN : 0069-5580

Référence électronique

François Déroche, «Histoire du Coran. Texte et transmission », L'annuaire du Collège de France [En ligne], 118 | 2020, mis en ligne le 01 avril 2021, consulté le 22 août 2022. URL : http://

journals.openedition.org/annuaire-cdf/15893; DOI : https://doi.org/10.4000/annuaire-cdf.15893 


\title{
HISTOIRE DU CORAN. TEXTE ET TRANSMISSION
}

\author{
François DÉROCHE \\ Membre de l'Institut (Académie des inscriptions et belles-lettres), \\ professeur au Collège de France
}

Mots-clés : Coran, texte, transmission, islam, religion, art, pouvoir, culture

La série de cours «Écrire le Coran $\left(\mathrm{V}^{\mathrm{e}}-\mathrm{X}^{\mathrm{e}}\right.$ siècle). Religion, art et pouvoir » est disponible en audio et/ou en vidéo sur le site internet du Collège de France (https:// www.college-de-france.fr/site/francois-deroche/course-2017-2018.htm), ainsi que le colloque " Le Coran dans l'histoire culturelle et intellectuelle de Fusțāț entre le vıle et le $X^{e}$ siècle » (https://www.college-de-france.fr/site/francois-deroche/symposium-20172018.htm).

\section{ENSEIGNEMENT}

\section{COURS - ÉCRIRE LE CORAN (VII ${ }^{e} X^{e}$ SIÈCLE). RELIGION, ART ET POUVOIR}

En l'an 1000, un copiste qui compte parmi les grands maîtres de la calligraphie arabe, 'Alī b. Hilāl, plus connu comme Ibn al-Bawwāb, achevait la transcription d'une copie du Coran qui est de nos jours conservée à la Chester Beatty Library de Dublin. Le manuscrit lui-même ne nous retiendra guère, sauf pour un point : son écriture. Celle-ci est en définitive relativement proche de celle que nous trouvons sur des exemplaires imprimés contemporains. En d'autres termes, un lecteur n'aurait de nos jours guère de difficulté à lire cet exemplaire vieux d'un millénaire. À titre de comparaison, un manuscrit latin de la même époque donnerait sans aucun doute plus de fil à retordre à celui qui, sans avoir reçu une formation de paléographie latine, tenterait de le déchiffrer.

Si le temps semble s'être arrêté à une date très haute pour l'écriture arabe, les premiers siècles de l'islam ont connu un développement graphique qui retient l'attention parce qu'il concerne presque exclusivement, dans le domaine des manuscrits, les copies du Coran qui, par ailleurs, ont été produites en quantités considérables. Pour l'étudier, les chercheurs disposent par chance d'une abondante documentation dont la recherche a cependant longtemps négligé l'importance tant 
pour l'histoire du texte coranique que pour la connaissance de la civilisation de l'islam au cours des quatre premiers siècles. Les manuscrits de cette période apparaissent souvent comme des feuillets isolés et, partant, comme des témoignages dont l'exploitation est difficile. Or cette dispersion est souvent récente et concerne principalement des exemplaires du Coran qui étaient en fait entreposés sans soin particulier dans un même lieu, à l'abri des souillures qui auraient pu affecter le nom divin. Nous connaissons quatre importants dépôts qui ont joué un rôle central pour notre compréhension de cette histoire et qui ont été « découverts » successivement depuis la fin du XVIII ${ }^{\mathrm{e}}$ siècle.

Avant que des savants et collectionneurs européens ne s'avisent de leur existence, ces dépôts étaient sans doute connus de ceux qui vivaient à proximité : nous le savons grâce à des feuillets isolés ou des morceaux de manuscrits qui ont été en possession de bibliophiles musulmans intéressés par ces écritures anciennes qu'ils mettaient peut-être en relation avec les copies du Coran réalisées sur l'ordre du calife 'Uthmān ou plus généralement par des Compagnons de Muhammad ${ }^{1}$. Un commerce de « reliques » a sans doute exploité ponctuellement ces dépôts, mais il a également concerné des manuscrits isolés dans différentes parties du monde musulman. Les grands corans «à douze lignes », comme ceux du Caire et de Tachkent ${ }^{2}$, sont d'excellents exemples de cette réutilisation de copies anciennes. Les modes de préservation de ces manuscrits et fragments diffèrent sensiblement de ce qui vaut pour le reste du patrimoine manuscrit arabo-musulman, un point à prendre en considération dans notre évaluation de ce patrimoine.

Dans l'Europe chrétienne, antérieurement au développement d'études orientalistes dignes de ce nom, les fragments ou manuscrits appartenant à l'ensemble qui nous intéressent sont restés rares jusqu'au XVII ${ }^{\mathrm{e}}$ siècle. Cela s'explique d'abord en grande partie par le fait que ce type de copies coraniques avait cessé d'être en usage dès le $\mathrm{XI}^{\mathrm{e}}$ siècle et que nombre d'entre eux se trouvaient renfermés dans les dépôts dont il a été question. De ce fait, les possibilités d'acquisition par les voies classiques se trouvaient considérablement réduites. À partir du XVII ${ }^{\mathrm{e}}$ siècle, des savants européens signalent l'existence de rares manuscrits qu'ils appellent «coufiques» dans différentes collections européennes, mais l'information demande à être prise avec précaution. Le terme « coufique », dont on connaît la fortune dans les études araboislamiques, a pu en effet être appliqué de manière erronée, par les Occidentaux qui s'intéressaient aux manuscrits arabes aux $\mathrm{XVII}^{\mathrm{e}}$ et $\mathrm{XVIII}{ }^{\mathrm{e}}$ siècles, à des spécimens qui, de nos jours, seraient définis de manière différente. Quoi qu'il en soit, l'entrée dans des collections européennes de copies coraniques anciennes est bien attestée au XVII ${ }^{\mathrm{e}}$ siècle. C'est en effet en 1627 qu'un capitaine ou négociant danois, Frederik Bockwold, acquiert au Caire dans des conditions obscures un petit groupe de

1. Voir par exemple J.-M. MouTON, « De quelques reliques conservées à Damas au Moyen Age. Stratégie politique et religiosité populaire sous les Bourides », Annales islamologiques, vol. 27, 1993, p. 247-254.

2. Manuscrit du Caire: T. AltikUlAÇ (dir.), Al-mușhaf al-šarīf (en arabe). Al-muṣhaf al-sharif attributed to 'Uthman bin 'Affan (The copy at al-Mashhad al-Husayni in Cairo), 2 vols, Istanbul, IRCICA, 2009 [1430]. Manuscrit de Tachkent : F. DÉROCHE, « Twenty leaves from the Tashkent Koran », in S. BLAIR et J.M. BLOOM (dir.), God Is Beautiful and Loves Beauty. The Object in Islamic Art and Culture, New Haven/Londres, Yale University Press, 2013, p. 57-77 et bibliographie. 
manuscrits qui passent rapidement dans les collections royales ${ }^{3}$. En France, le coran dit «de Charlemagne» est peut-être également devenu la propriété d'un certain Cochu vers cette même époque - il fait en tout cas partie des collections royales à la fin du XVIII ${ }^{\mathrm{e}}$ siècle ${ }^{4}$. Malheureusement, son histoire est mal connue : il est clair que cette copie du Coran n'a pas été offerte par Harūn al-Rashīd à Charlemagne dans la mesure où un musulman ne remettrait pas en principe le texte révélé entre les mains d'un non-musulman, fût-il Charlemagne lui-même. De plus, l'étude paléographique invite plutôt à reconnaître dans cette copie un exemplaire du $\mathrm{IX}^{\mathrm{e}}$ siècle.

Les cinq manuscrits que Bockwold avait acquis sont, nous le savons maintenant, des volumes qui appartenaient à des copies en plusieurs tomes qui se trouvaient dans le premier des dépôts évoqués plus haut. Nous pouvons en déduire que, dès cette époque au moins, des habitants de Fusțâț avaient commencé à vendre à des Européens, amateurs d'antiquités ou de curiosités, des fragments plus ou moins importants de manuscrits coraniques, soit directement, soit en passant par des intermédiaires. Ils les retiraient certainement d'une pièce donnant sur la cour de la plus ancienne mosquée d'Égypte, celle que 'Amr b. al-'Aṣ avait fondée en 643 à Fusțāț, capitale de l'Égypte jusqu'à la fondation du Caire. Un peu plus tard, Friderich Christian von Haven, un membre de l'expédition de Carsten Niebuhr, acquit lors de son séjour au Caire entre novembre 1761 et août 1762 un feuillet de coran qui fut reproduit dans le Voyage en Arabie; le manuscrit d'où il fut pris était conservé dans la bibliothèque de la mosquée al-Azhar et était attribué au calife 'Umar, mais il provenait vraisemblablement lui-aussi de Fusțạt ${ }^{5}$. Ces transactions restaient cependant d'une ampleur limitée dans la mesure où elles étaient susceptibles de mettre l'une ou l'autre des parties impliquées dans une situation délicate. Niebuhr rapporte que von Haven dut déployer beaucoup d'efforts et payer grassement son maître d'arabe, qui avait accès à la bibliothèque d'al-Azhar, pour obtenir ce feuillet. Quant à Ulrich Seetzen, un voyageur allemand du début du XIX ${ }^{\mathrm{e}}$ siècle, il se plaint, lors de sa visite de janvier 1809, de la «bigoterie» des femmes qui se trouvaient

3. Manuscrits Copenhague, Bibliothèque royale, cod. Arab. XXXVI à XXXVIII, XL et XLI (Codices orientales bibliotheca regia hafniensis... Pars altera, codices hebraicos et arabicos continens, Hafniæ, 1851, p. 40-43 ; aussi J.G.C. ADLER, Descriptio codicum quorumdam cuficorum partes Corani exhibentium in Bibliotheca regia hafniensi et ex iisdem de scriptura Arabum observationes nova, Pramittitur disquisitio generalis de arte scribendi apud Arabes ex ipsis auctoribus arabicis adhuc ineditis sumta, Altona, 1780).

4. F. DÉROCHE, Catalogue des manuscrits arabes, $2^{\mathrm{e}}$ partie : Manuscrits musulmans, t. I, 1 : Les Manuscrits du Coran. Aux origines de la calligraphie coranique, Paris, Bibliothèque nationale, 1983, p. 73 ; id., La Voix et le calame. Les chemins de la canonisation du Coran, Paris, Collège de France/Fayard, coll. «Leçon inaugurale», vol. 255, 2016 ; édition électronique : Paris, Collège de France, 2016, DOI : 10.4000/books.cdf.4761, https://books. openedition.org/cdf/4761, fig. 5.

5. C. NiEBuHR, Description de l'Arabie d'après les observations et recherches faites dans le pays même, Copenhague, Chez Nicolas Möller, 1773, pl. 4 et 5. Le feuillet est actuellement conservé au Musée national de Copenhague (cf. S. Rasmussen, Carsten Niebuhr und die Arabische Reise 1761-1767, Heide in Holstein, 1986, p. 96, n 22). J.-J. Marcel a suggéré ce rapprochement (Paléographie arabe ou Recueil de mémoires sur différents monumens lapidaires, numismatiques, glyptiques et manuscrits présentant des inscriptions koufiques et karmatiques, dans tous les genres de caractères employés par les anciens Arabes, $\mathrm{I}^{\mathrm{re}}$ partie, Paris, 1828, p. 8). 
dans la mosquée de 'Amr et auprès desquelles il tenta en vain d'acheter quelques feuillets : elles lui objectèrent que les manuscrits étaient waqf et lui racontèrent que plusieurs personnes, dont des mamelouks, en avaient pris, mais les avaient rapportés parce qu'ils avaient perdu la tranquillité à la suite du vol qu'ils avaient commis ${ }^{6}$.

La situation avait pourtant changé avec l'expédition d'Égypte. Au cours de cette dernière, ceux qui cherchaient à acquérir des manuscrits, y compris des manuscrits coraniques, purent le faire dans des conditions beaucoup plus favorables que leurs prédécesseurs. Parmi les membres scientifiques de l'expédition figurait un jeune arabisant, Jean-Joseph Marcel, qui avait pour fonctions de s'occuper de l'imprimerie dont Bonaparte avait fait embarquer le matériel sur un des navires. Une fois les premiers succès remportés et les Français installés au Caire, Marcel allait mettre à profit à la fois ses connaissances d'arabe et sa position pour collecter des manuscrits. Dans les souvenirs qu'il a laissés, il évoque surtout l'épisode du sauvetage d'un coran de très grandes dimensions, arraché aux flammes de l'incendie de la mosquée d'al-Azhar ${ }^{7}$. Par d'autres sources, nous savons que son intérêt pour les livres anciens ne s'était pas arrêté là : en tant que membre de l'Institut d'Égypte, il a dû, au sein de sa Commission des sciences et des arts, prendre part aux acquisitions des manuscrits qui seront déposés en 1803 à la Bibliothèque nationale. La Bibliothèque nationale de Russie à Saint-Pétersbourg abrite même un fonds qui porte son nom et regroupe les fragments achetés par Marcel en Égypte. Comment et où en fit-il l'acquisition ? Nous ne disposons pas d'information plus précise à ce sujet qu'une note de sa main dans laquelle il déclare qu'il s'agit de fragments "recueillis dans la mosquée d'Amrou au Vieux Kair (sic) en Égypte ${ }^{8}{ }$. Ils sont donc les premiers spécimens du dépôt de Fusțăț dont nous ayons connaissance de manière directe. Lorsque, pressée par les Anglo-Ottomans, la garnison française du Caire dut signer la convention du 27 juin 1801, une clause prévit que les Français et notamment les savants eussent le droit d'emporter leurs possessions avec eux ; la collection sortit ainsi d'Égypte pour rejoindre la France ${ }^{9}$.

De son vivant, Marcel ne se défit pas de cet ensemble ; après sa mort, il fut acquis de son héritière, une dame Desnoyers, par le gouvernement russe grâce à l'entremise du diplomate et orientaliste Nicolas V. Khanykov et passa ainsi de Paris à SaintPétersbourg. Des notes plus récentes sur les papiers de Marcel sont dues à Victor Langlois qui se chargea de mettre en ordre les fragments et d'en faire l'inventaire avant qu'ils ne gagnent la Bibliothèque publique en $1864^{10}$. Longtemps, Marcel avait caressé l'espoir de publier une paléographie arabe dans laquelle il aurait tiré parti des fragments coraniques de sa collection, mais il n'alla pas plus loin qu'un prospectus où il donnait les grandes lignes de son projet.

6. F. KRuSE (dir.), Ulrich Jasper Seetzen's Reisen durch Syrien, Palästina, Phönicien, die Transjordan-Länder, Arabia Petraea und Unter-Aegypten, t. 3, Berlin, G. Reimer, 1855, p. 389.

7. J.-J. MARCEL, Contes du Cheykh êl-Mohdy, t. 1, Paris, Imprimerie F. Locquin, 1832, p. 413-414.

8. Dossier Saint-Pétersbourg, BNR, Marcel 132, f. 1 («Lettre à M. Étienne Quatremère... »).

9. H. Laurens, avec la collab. De C.G. Gillispie, J.-C. Golvin et C. Traunecker, L'Expédition d'Égypte 1798-1801, Paris, Armand Colin, 1989, p. 319.

10. Otchet' Imperatorskoj publichnoj biblioteki za 1864 goda, Saint-Pétersbourg, 1865, p. 22-24 ; Otchet' Imperatorskoj publichnoj biblioteki za 1865 goda, Saint-Pétersbourg, 1866, p. 51 ( $\mathrm{n}^{\circ}$ 5) ; O.V. VASILYEVA, «Oriental manuscripts in the National Library of Russia », Manuscripta Orientalia, vol. 2, no 2, 1996, p. 20. 
Une idée presque identique se retrouve sous la plume de Jean-Louis Asselin de Cherville qui déclarait dans une lettre de 1814 avoir rassemblé

[...] une collection considérable de feuilles du Coran en caractères coufiques sur peau de gazelle depuis les premiers siècles de l'islamisme, jusqu'à l'époque où ces caractères ont cessé d'être en usage pour former une paléographie arabe. Je possède [ajoutait-il,] plusieurs feuilles du plus ancien manuscrit de cette espèce qui soit connu ${ }^{11}$.

Asselin de Cherville avait entamé sous la Révolution des études orientales et avait ainsi suivi les cours d'Antoine-Isaac Silvestre de Sacy. Il avait obtenu du ministère des Affaires étrangères un poste de drogman au Caire qu'il rejoignit en 1806, soit deux ans avant que ne fût publié le Mémoire sur l'origine et les anciens monuments de la littérature parmi les Arabes de Silvestre de Sacy. Certaines des conclusions de son maitre sur les écritures anciennes, cependant, et plus spécialement un passage du Fihrist, pouvaient avoir déjà été connus de lui ${ }^{12}$; il n'est pas non plus exclu qu'il ait pu examiner à Paris la collection de Marcel. Ces informations l'ont-elles guidé dans l'acquisition des fragments qui sont de nos jours à la Bibliothèque nationale de France ? Ce qu'il écrivait à ce propos se rapporte sans doute au Codex Parisinopetropolitanus dont le fragment le plus important figurait dans sa collection, mais on peut également penser au Codex omeyyade de Fusțât ${ }^{13}$. L'espoir que nourrissait Asselin de Cherville de voir son activité scientifique pleinement reconnue ne se réalisa toutefois pas et il mourut au Caire en 1822.

La collection de manuscrits d'Asselin de Cherville arriva à Marseille en 1825 et ses héritiers cherchèrent à la vendre. Après avoir pensé d'abord trouver un acquéreur en Grande-Bretagne, ils se mirent d'accord avec la Bibliothèque royale. Une note, dont l'auteur, anonyme, pourrait bien être Joseph Toussaint Reinaud, conservateur des manuscrits orientaux, évaluait l'intérêt de la collection d'Asselin de Cherville, peut-être avant que la vente n'ait été conclue ${ }^{14}$. Elle ne manquait pas d'attirer l'attention du destinataire (ce pourrait être le directeur de la Bibliothèque, Letronne) sur les fragments en caractères coufiques qui offraient de nouveaux moyens d'étude aux amateurs de paléographie arabe. L'acquisition fut conclue en 1833 et eut probablement pour conséquence indirecte la vente à la bibliothèque de SaintPétersbourg des feuillets de Marcel : la bibliothèque jugea sans doute posséder déjà une collection suffisamment riche pour avoir besoin d'acquérir ces fragments qui lui furent proposés après le décès de Marcel.

Les bibliothèques de Paris et de Saint Pétersbourg n'étaient toutefois ni les seules ni même les premières en Europe à conserver des feuillets provenant de Fusțât : le voyageur allemand mentionné précédemment, Seetzen, avait en effet acheté en 1808,

11. J.-L. Asselin De Cherville, « Lettre de M. J.-L. Asselin de Cherville, Agent du Consulat général de France, au Caire, à M. Dacier, Secrétaire perpétuel de la troisième Classe de l'Institut », Magasin encyclopédique, vol. 3, 1815, p. 88.

12. Voir son «Mémoire sur l'origine et les anciens monuments de la littérature parmi les Arabes », Mémoires de littérature tirés des registres de l'Académie royale des inscriptions et belles-lettres, t. 50, 1808, p. 247-348.

13. F. DÉroche, La Transmission écrite du Coran dans les débuts de l'islam. Le Codex Parisino-petropolitanus, Leyde/Boston, Brill, coll. « Texts and studies on the Qur'ān », vol. 5, 2009 ; id., Qur'ans of the Umayyads. A First Overview, Leyde/Boston, Brill, 2014, p. 75-97.

14. Manuscrit Paris, BNF, NAF 5441. 
pour le compte de la collection de Gotha, un petit lot qui y parvint dès 1810 et fut l'objet d'une publication en deux étapes ${ }^{15}$. L'histoire du fonds de Fusțāt est en effet caractérisée par sa dispersion progressive qui se poursuivit tout au long du XIX ${ }^{\mathrm{e}}$ siècle - et même au-delà. La décision fut bien prise par les autorités égyptiennes de transférer à la bibliothèque khédiviale ce qui restait du fonds à la mosquée de 'Amr, mais il semble bien que des antiquaires avaient pris les devants. Comment expliquer autrement l'acquisition par un égyptologue britannique du manuscrit Or. $2165^{16}$, ou encore les petites collections de Chicago ou de la Vaticane ${ }^{17}$, pour ne pas parler de la vente aux enchères à Rennes en 2011 d'un important lot de feuillets qui était en la possession d'un antiquaire d'origine grecque qui avait de bons contacts en Égypte ${ }^{18}$. À la différence de Marcel et d'Asselin de Cherville qui ont privilégié l'achat de portions substantielles de manuscrits, les antiquaires trouvaient plus d'intérêt à vendre isolément des feuillets, ce qui a accru leur dispersion et par suite la difficulté du remembrement.

Des différents dépôts de mosquée, celui de Fusțâț est le mieux connu en ce qui concerne les contenus. Les fragments acquis par Asselin de Cherville ont en effet été étudiés dès le XIX ${ }^{\mathrm{e}}$ siècle par Michele Amari, dont les notices, bien que succinctes, ont été intégrées au catalogue de Slane ${ }^{19}$. D'autres parties de la collection, tels les fragments qui sont maintenant conservés à Gotha, Chicago ou au Vatican, ont été décrits, des spécimens des premiers ayant même été reproduits sous forme de facsimilé dès 1844 par Johann Heinrich Möller ${ }^{20}$; d'autres sont moins bien connues, mais accessibles. Le fonds de Dār al-kutub au Caire, où devraient se trouver les feuillets de la mosquée de Fusțāt qui furent transférés au XIXe siècle à la Bibliothèque khédiviale, est celui à propos duquel nos informations sont les plus minces.

De nombreux savants, par exemple Yusuf Éche, étaient convaincus qu'il ne restait rien de l'ancienne collection de la Grande Mosquée de Damas : dans son histoire des bibliothèques du Proche-Orient à l'époque médiévale, Éche arrivait à la conclusion que les livres les plus anciens présents dans la Grande Mosquée avaient tous été détruits par un incendie en $1068^{21}$.

15. J.H. MÖLlER, Paläographische Beiträge aus den herzoglichen Sammlungen in Gotha, Eisleben, G. Reichardt, 1844 ; W. PERTSCH, Die orientalischen Handschriften der herzoglichen Bibliothek zu Gotha, t. III : Die arabischen Handschriften, vol. 1, Gotha, Friedr. Andr. Perthes, 1878 , p. 376-396 [n $\left.{ }^{\text {os }} 427-464\right]$.

16. F. DÉRoche et S. NojA NosedA, Sources de la transmission manuscrite du texte coranique, I : Les Manuscrits de style higâzî, t. II, 1 : Le Manuscrit Or. 2165 (f. 1 à 61) de la British Library, Lesa, Fondazione Ferni Noja Noseda/Studi Arabo Islamici, 2001.

17. Pour Chicago, voir N. Аввотт, The Rise of the North Arabic Script and Its Kur'anic Development, Chicago, University of Chicago Press, 1939 ; pour le Vatican : G. LEVI DELLA VIDA, Frammenti coranici in carattere cufico nella Biblioteca Vaticana, Vatican, Biblioteca Apostolica Vaticana, 1947.

18. M.C. DAVID (Cabinet d'expertise), Collection d'un Antiquaire de la première moitié du XX ${ }^{e}$ siècle, Rennes Enchères, 19 septembre 2011.

19. W. MC GUCKIN DE SLANE, Catalogue des manuscrits arabes, Paris, Imprimerie nationale, 1883-1895, p. 87-117.

20. J.H. MÖLLER, op. cit.

21. Y. ECHÉ, Les Bibliothèques arabes publiques et semi-publiques en Mésopotamie, en Syrie et en Égypte au Moyen Âge, Damas, Institut français de Damas, 1967, p. 202. 
Il s'avère toutefois qu'une grande collection, analogue en tous points à celle de Fusțāt, a longtemps été déposée dans la mosquée des Omeyyades. L'histoire de sa découverte par les voyageurs occidentaux et les orientalistes n'est pas complètement élucidée. La première information claire dont on ait disposé en Europe sur le gisement de Damas date de 1862 : cette année-là, la Bibliothèque royale de Berlin fit l'acquisition de la deuxième collection de Gottfried Wetzstein ${ }^{22}$. Ce dernier, qui fut consul de la Prusse à Damas entre 1848 et 1862, était orientaliste et un collectionneur de manuscrits avisé : il disposait d'un réseau d'intermédiaires dans toute la région, mais il n'existe pas de renseignement précis sur la façon dont il avait eu vent de l'existence du dépôt de manuscrits coraniques anciens de la Grande Mosquée, ni sur la manière dont il réussit à en acquérir de nombreux fragments ${ }^{23}$.

Les acquisitions par les Occidentaux avaient toutefois peut-être commencé avant l'époque de Wetzstein : un volume factice de la Bibliothèque nationale de France, Arabe 6140, faisait partie de l'importante collection que l'érudit oriental français Charles Schefer forma pendant les années qu'il passa dans l'Empire ottoman en tant que fonctionnaire du ministère français des Affaires étrangères de 1843 à 1857 . Sa position était donc en partie similaire à celle de Wetzstein. Des folios coraniques de différentes périodes constituent le contenu d'Arabe 6140. Ceux sur parchemin ont été presque certainement pris dans le dépôt à Damas. Nous en avons la certitude pour certains d'entre eux, notamment pour les f. 1-4, mais le reste est probablement de la même origine. Il n'y a pas d'indice quant à la date de l'achat : Schefer les a-t-il acquis alors qu'il servait à Beyrouth ou à Jérusalem, au début de sa carrière entre 1843 et 1846, peu avant l'arrivée de Wetzstein à Damas ? Ou les a-t-il obtenus quand il était à Constantinople, c'est-à-dire après 1849 ?

Le monde savant fut vite informé que Wetzstein avait acheté des fragments puisque la collection de Berlin est mentionnée dans la Geschichte des Qorâns publiée par Theodor Nöldeke en $1860^{24}$. Après cette date, les voyageurs à Damas à la recherche de manuscrits devaient savoir qu'il était possible d'acquérir de vieux fragments coraniques. La même situation s'est produite au Caire : après la visite de Marcel à la mosquée de 'Amr, la dispersion des fragments de Fusțât au XIX' siècle a été intense. Peu après le départ de Wetzstein de Damas, deux Anglais, Edward Henry Palmer (qui devint plus tard professeur d'arabe à Cambridge) et Charles F. Tyrwhitt Drake, entreprirent un voyage au Proche-Orient avec le soutien du Fonds d'exploration de la Palestine. Venus de Pétra et de Jérusalem, ils s'arrêtèrent à Damas en 1870 pour rentrer en Grande-Bretagne par Constantinople ${ }^{25}$. Ils ont, semble-t-il, bénéficié de l'hospitalité du consul britannique à Damas, le célèbre capitaine Richard Burton, qui les a peut-être aidés dans la ville. Bien qu'il n'y ait aucune indication claire sur la provenance de chacune des pièces qui font maintenant partie de la collection de la

22. F. DÉROCHE, "The Quranic collections acquired by Wetzstein », in B. LIEBRENZ et C. RauCH (dir.), Manuscripts, Politics and Oriental Studies. Life and Collections of Johann Gottfried Wetzstein (1815-1905) in Context, Leyde/Boston, Brill, 2019, p. 92-115.

23. Ibid., p. 107, n. 64.

24. T. NÖLDEKE, Geschichte des Qorâns, Göttingen, Verlag der Dieterichschen Buchhandlung, 1860, p. 304.

25. Voir W. BESANT, The Life and Achievements of Edward Henry Palmer, Late Lord Almoner's Professor of Arabic in the University of Cambridge and Fellow of Saint John's College, Londres, J. Murray, 1883, p. 109. 
bibliothèque de l'université de Cambridge, les preuves que j'ai recueillies indiquent de manière très consistante une origine damascène, à l'exception d'au moins un fragment acquis à Jérusalem par Palmer et Tyrwhitt Drake ${ }^{26}$. La collection fut achetée par la bibliothèque de l'université de Cambridge en 1874.

Les manuscrits et papiers usés étaient conservés à l'intérieur du Bayt al-Māl, dans la cour de la Grande Mosquée. Ce détail n'a pas été connu en Occident avant les années 1870. Une première indication peut être trouvée sur un plan dessiné par l'architecte français Jules Bourgoin, qui a visité Damas en 1874 ou 1875. Sur son dessin, à côté du Bayt al-Māl dans la cour de la mosquée, nous pouvons voir une légende -» Bibliothèque »-, une désignation qui coïncide avec une appellation locale dont on trouve la trace par la suite: Khizāna ${ }^{27}$. Les archives ottomanes donneront sans doute plus de renseignements sur la façon dont l'accès au dépôt s'est fait dans la seconde moitié du XIX ${ }^{\mathrm{e}}$ siècle. D'après cette source, il apparaît que, du point de vue officiel, le bâtiment de la cour de la Grande Mosquée avait été ouvert vers 1890, mais que les autorités l'avaient fait fermer. En mars 1900, un fonctionnaire de l'Instruction publique en sollicitait l'ouverture à la suite d'une requête de l'ambassade d'Allemagne qui intervenait à la demande du célèbre bibliste Hermann von Soden 28.

La situation a sans doute été plus complexe. En 1898, l'empereur Guillaume II visita Damas lors d'une visite d'Etat. Cela constitua une occasion extraordinaire pour deux chercheurs allemands d'accéder officiellement au dépôt du Bayt al-Māl. Bruno Violet, qui y a passé plus de temps, a décrit dans l'introduction d'un article sur sa trouvaille la plus importante, une traduction arabe des Psaumes en lettres grecques, comment il a pu travailler pendant un mois entier, se dépêchant de voir le plus grand nombre possible de fragments. Selon ses propos, il y avait alors beaucoup de rumeurs qui courraient sur la collection et on laissait entrer les visiteurs sous couvert du secret (ce qui indique que l'accès était possible en dépit de la «fermeture » officielle) ${ }^{29}$. Dans l'édicule, les fragments étaient entassés jusqu'à la taille d'un homme. Violet a vu une majorité de fragments arabes, comme on pouvait s'y attendre, mais il mentionne aussi le grec, le syriaque, le samaritain et même le vieux français et le copte. Pendant un moment, il fut aidé dans sa tâche par un collègue, Bernhard Moritz.

Comme ce dernier était à l'époque directeur de la Bibliothèque khédiviale au Caire, il peut être responsable de la présence dans la bibliothèque du Caire d'un juz' du Coran avec une reliure qui fut publiée en 1929 par Adolf Grohmann et qui contient une waqfiyya à la Grande Mosquée de Damas ${ }^{30}$. Il faut souligner que certains manuscrits ont circulé même après avoir été constitués en waqf dans un lieu d'où, en principe, ils ne devaient pas sortir. Dans la mosquée de 'Amr à Fusțâț se trouvent deux volumes venus de Tarse - sur la côte sud de la Turquie moderne - et

26. Cambridge, CUL, MS Add. 1130.

27. Paris, École nationale supérieure des Beaux-Arts, Musée, no 8564.

28. Je remercie très vivement $\mathrm{M}$. Edhem Eldem de m'avoir communiqué cette référence ainsi que celles qui figurent plus loin en n. 36.

29. B. Violet, «Ein zweisprächiges Psalmfragment aus Damaskus », Orientalistische Literatur Zeitung, vol. 4, 1901, p. 384-385.

30. T.W. ARnOLD et A. Grohmann, The Islamic Book. A Contribution to Its Art and History from the VII-XVIII Century, [Paris/New York], Pegasus Press/Harcourt Brace \& Co, 1929, p. 46-47, pl. 22 b-c (manuscrit Le Caire, Dār al-kutub, Mașāḥif 192). 
un autre de $\operatorname{Tyr}^{31}$; des cas similaires sont attestés dans la collection de Damas ellemême, à commencer par le coran d'Amājūr, dont des fragments ont été en possession de Wetzstein et de Palmer ${ }^{32}$. En tout cas, les témoignages de Violet et des archives ottomanes coïncident pour établir que, quelques années après la destruction de la mosquée des Omeyyades par un incendie en 1893, des manuscrits étaient encore conservés dans le Bayt al-Māl dans la cour de la mosquée ; ils ne s'accordent pas sur les conditions de conservation, le fonctionnaire ottoman mentionnant des caisses en fer alors que le savant allemand décrivait une situation similaire à celle de Fusțāṭ.

Un scénario de dispersion des contenus similaire à celui de Fusțāt avait débuté à Damas vers la fin du XIX ${ }^{\mathrm{e}}$ siècle. Un fragment de quelques feuillets qui est conservé à la Chester Beatty Library de Dublin et qui porte un acte de waqf en faveur de la Grande Mosquée de Damas est associé aux agissements d'un collectionneur de la fin du XIX ${ }^{\mathrm{e}}$ et du début du XX ${ }^{\mathrm{e}}$ siècle, Fredrick Robert Martin. Né en 1868, ce diplomate suédois se rendit en Égypte, au Proche-Orient et en Anatolie, en 1895 et 1896, avant de rejoindre l'ambassade de Suède à Constantinople en 1904, où il servit de drogman jusqu'en 1908. Il collectionnait des manuscrits pendant cette période, et semble avoir éprouvé peu de scrupules quand il s'agissait d'agrandir sa collection. Comme ce fut le cas avec la collection Schefer, nous ne savons pas où Martin a acquis les feuillets que j'ai mentionnés il y a un instant. Avaient-ils déjà atteint Istanbul avant l'arrivée de Martin en 1904 et avant son départ en 1908 ? Ils auraient en effet pu être amenés de Damas à l'époque ottomane, comme cela est arrivé pour des fragments de Fusțât, et avoir été mis en vente sur place. Mais nous ne pouvons pas exclure la possibilité que Martin ait mis la main sur eux plus tôt alors qu'il voyageait au Proche-Orient.

Il est possible que les antiquaires locaux aient commencé à vendre du matériel obtenu à partir du dépôt. Cela pourrait expliquer pourquoi un autre manuscrit damascène, ms. Or. oct. 1819, atteignit la Staatsbibliothek de Berlin en 1918 ou 1919 sans passer par Wetzstein. Sa provenance peut être établie grâce à une waqfiyya qui nomme la Grande Mosquée de Damas, et la reliure publiée par Max Weisweiler est tout à fait conforme à ce que nous savons de la production locale au $\mathrm{IX}^{\mathrm{e}}$ siècle. $\mathrm{La}$ bibliothèque acheta ce volume à la femme de Julius Loytved-Hardegg ${ }^{33}$. Ce dernier a été vice-consul à Haïfa, puis consul à Damas pendant la Première Guerre mondiale. Où et comment a-t-il obtenu ce volume ? Comme nous le verrons, nous pouvons exclure une acquisition directe du dépôt du Bayt al-Mâl. Faisait-il partie d'un groupe de fragments qui en étaient sortis auparavant et entrés dans les circuits des antiquaires ? Un informateur m'a dit, par exemple, que Jean Sauvaget avait acheté quelques feuillets à Damas dans les années 1940. D'autres, provenant du célèbre coran d'Amājūr, se trouvent maintenant dans une collection saoudienne et pourraient avoir été acquis de la même manière ${ }^{34}$. En fait, nous ne pouvons pas exclure la

31. F. DÉROCHE, op. cit., p. 86 (Tyr), 91 et 126 (Tarse).

32. Manuscrit Cambridge, CUL MS Add. 1116 ; un feuillet resté en possession de Wetzstein a rejoint par la suite les collections de la Staatsbibliothek (Ms. Or. Quart. 1208, $\mathrm{n}^{\mathrm{o}}$ II, f. 1). Voir aussi A. GEORGE, «The Geometry of the Qur'an of Amajur: A Preliminary Study of Proportion in Early Arabic Calligraphy », Muqarnas, vol. 20, 2003, p. 115 et bibliographie.

33. M. WeISWEILER, Der islamische Bucheinband des Mittelalters nach Handschriften aus deutschen, holländischen und türkischen Bibliotheken, Wiesbaden, O. Harrassowitz, coll. « Beiträge zum Buch- und Bibliothekenwesen », vol. 10, 1962, p. 92 et fig. 1.

34. Voir plus haut. 
possibilité que les fragments achetés par Schefer ou Martin, ou même le feuillet que Palmer et Tyrwhitt Drake avaient trouvé à Jérusalem, soient passés par les mains d'antiquaires locaux.

Les différents fragments mentionnés jusqu'à présent ne représentent toutefois qu'une infime partie de ce qui a été conservé du dépôt de Damas. La majorité est aujourd'hui conservée à Istanbul. Comment est-il arrivé dans cette ville ? On a écrit que le transfert de Damas à Istanbul était une conséquence de l'incendie de 1893. Mais le récit de Violet et les archives ottomanes montrent que la collection était toujours à Damas en 1898. Selon certaines sources turques, la collection de Damas atteignit Istanbul en 1911 et fut d'abord remise au musée de Topkapı Saray1, avant d'être transférée au musée des Evqaf, plus tard rebaptisé Türk ve İslam Eserleri Müzesi (musée des Arts turc et islamique), où elle est maintenant conservée ${ }^{35}$. Cependant, différents documents dans les archives du Başbakanlık indiquent que la collection a été retirée de Damas pendant la Première Guerre mondiale ${ }^{36}$. Les premiers, datés de 1916, font état des préoccupations de l'administration ottomane pour mettre à l'abri les fragments «se trouvant dans les sous-sols de la cour de la mosquée des Omeyyades » en les transférant à Istanbul avec le concours de l'armée. Le transport eut lieu l'année suivante, comme l'indiquent des dépêches de janvier et février 1917: la plus haute autorité religieuse, le Sheykhülislam Mūsā Kāẓım Efendi, supervisa l'opération qui fut été menée à bien avec l'aide de la quatrième armée ottomane sous le commandement de Cemal Pacha. Une fois les fragments arrivés à Istanbul, le musée de Topkapı Sarayı en a recueilli quelques-uns : selon un conservateur du musée des Arts turc et islamique, les articles les plus beaux ont été sélectionnés par le personnel de l'ancien musée. En travaillant sur les plus anciennes copies du Coran conservées à la bibliothèque de Topkapı Sarayı, j'ai pu effectivement identifier dans la série Emanet Hazinesi quelques volumes qui faisaient partie d'exemplaires du Coran en plusieurs volumes qui se trouvaient autrefois à Damas.

La plupart des folios sont toutefois conservés au musée des Arts turc et islamique où la collection est demeurée dans l'état où elle était au début du $\mathrm{XX}^{\mathrm{e}}$ siècle. C'est clairement une geniza puisqu' on y trouve aussi des documents et des amulettes, des livres et des certificats, et des textes arabes et latins. Les manuscrits coraniques sont de loin les plus nombreux, mais des compositions littéraires ont également été conservées. Une bibliothèque où les livres étaient physiquement maintenus ensemble existait sans doute dans la mosquée à l'époque médiévale. À partir des indications qui sont parfois directement liées aux manuscrits coraniques, nous savons cependant que certains d'entre eux étaient disponibles dans divers points de la salle de prière, notamment des séries en plusieurs volumes qui étaient conservées dans des boîtes.

Parmi les dépôts de manuscrits anciens du Coran, celui de Damas est numériquement le plus important. Il est pourtant mal connu : certes, les deux collections vendues par Wetzstein en Allemagne ont été cataloguées dès le $\mathrm{XIX}^{\mathrm{e}}$ siècle, mais de manière assez sommaire. Il en va de même pour les fragments de Cambridge, bien que leur récente numérisation se soit accompagnée d'un effort

35. A. Serkander Demirkol et S. Kutluay, « About the Museum of Turkish and Islamic Art Qur'an collection », in A. SERKANDER DEMIRKOL, S. KuTluAY et S. ŞAHIN (dir.), The 1400 th Anniversary of the Qur'an. Museum of Turkish and Islamic Art Qur'an Collection, Istanbul, Antik A.Ş. Cultural Publications, 2010, p. 139-140.

36. Başbakanlık Osmanlı Arşivi, DH. ŞFR. 72/3, DH. ŞFR. 73/70 et DH. ŞFR. 73/73. 
de présentation des contenus. Du matériel qui a été transporté de Damas à Istanbul, seul celui qui a été déposé à la bibliothèque du musée de Topkapı Sarayı a fait l'objet d'une description très succincte ${ }^{37}$. En revanche, à l'exception de quelques manuscrits ou fragments qui ont fait l'objet d'un signalement, la masse des fragments conservés au musée des Arts turc et islamique attend qu'on en fasse une présentation scientifique.

Une troisième collection, similaire aux deux précédentes par son statut, celui d'un dépôt de manuscrits au rebut, fut découverte à la fin du XIX ${ }^{\mathrm{e}}$ siècle à Kairouan. On ne sait pas à quelle date une bibliothèque a été installée dans la mosquée elle-même. Les manuscrits qui faisaient partie de ce dépôt ne nous ont donné aucune information à ce sujet. Des savants locaux ou des voyageurs musulmans connaissaient toutefois leur existence. Dans les dernières années du XIII ${ }^{\mathrm{e}}$ siècle, al-'Abdarī a vu lors de son séjour à Kairouan les pièces remarquables :

Nous sommes entrés dans la mosquée et avons visité la bibliothèque : ils ont sorti pour nous de nombreux corans en écriture orientale, y compris certains complètement écrits avec de l'or; sur certains d'entre eux, il était écrit qu'ils étaient des waqf très anciens qui remontaient à l'époque de Sahnnūn et même avant lui. Parmi ces œuvres était également Muwațțā' d'Ibn al-Qāsim et d'autres. J'ai vu une copie complète du Coran, réunie entre deux planches de bois reliées, sans aucun signe d'usure, dans une écriture orientale admirablement équilibrée, longue de deux empans et large d'un et demi et ils nous ont dit qu'elle avait été envoyée au Maghreb par Uthmān ${ }^{38}$.

Le déclin de la ville et l'importance croissante de Tunis dans le domaine des études traditionnelles mirent presque en sommeil la bibliothèque, alors que celles de Tunis, surtout de la mosquée Zaytūna, se développèrent de manière notable, comme le confirment les sources arabes.

L'importance historique de la bibliothèque est alors découverte par les chercheurs vers la fin du XIX ${ }^{\mathrm{e}}$ siècle. En 1882, peu de temps après que la France eut imposé son protectorat à la Tunisie, une mission scientifique française composée des arabisants Octave Houdas et René Basset avait cherché à établir, entre autres, l'état des bibliothèques tunisiennes. Mais elle avait trouvé bien peu à Kairouan :

[...] c'est ainsi que nos recherches dans les principales mosquées, déclaraient Houdas et Basset, n'ont abouti qu'à la découverte de quelques feuillets dépareillés d'ouvrages de droit et quelques copies du Qorân servant à la prière ${ }^{39}$.

- Mais les auteurs ajoutaient :

[...] nous soupçonnons cependant qu'à l'approche de l'armée française, les fonctionnaires ecclésiastiques musulmans se hâtèrent de faire disparaître les livres qui avaient pu échapper aux dévastations antérieures et d'en enrichir leurs bibliothèques particulières ${ }^{40}$.

37. Voir F.E. Karatay, Topkapı Sarayı Müzesi Kütüphanesi arapça yazmalar kataloğu, vol. 1 : Kur'an, Kur'an ilimleri, Tefsirler. No. 1-2171, Istanbul, Topkap1 Saray1 Müzesi, 1962, passim.

38. AL-'ABDARĪ, Rihlala, édité par 'A.I. KARWĪ et R. AL-FAHHĀM, Damas, Dār Sa'd al-dīn li-1țibā'a, 1999, p. 59.

39. O. Houdas, R. BASSet et Lougarre, Mission scientifique en Tunisie (1882), Alger, Imprimerie de P. Fontana, 1884, p. 143.

40. Ibid. 
Il ne semble pas exclu que les manuscrits aient été dissimulés par crainte d'une confiscation par les Français, mais, comme nous le verrons, l'allégation de vol n'était pas justifiée - sauf peut-être pour certains manuscrits. En revanche, la publication par Houdas de feuillets caractéristiques de textes légaux de Kairouan dans son «Essai sur l'écriture maghrébine » représente une première information sur ce fond - même si la première publication est venue d'ailleurs ${ }^{41}$. Nous ne savons pas comment Houdas acheta ces feuillets ; en tout cas, il en fit don aux bibliothèques d'Alger et de l'École des langues orientales vivantes de Paris.

En 1897, le voyageur égyptien Muhammad Bayrām Bey, au cours d'une visite en Tunisie, vit à Kairouan les fragments de la bibliothèque médiévale. Leur mauvais état le poussa à en faire état dans un journal du Caire, le Muqtataf, et à demander au gouvernement tunisien de prendre des mesures de conservation :

Dans cette pièce il y a deux grands placards pleins de paquets de papier, faits pour la majorité des pages détachées mélangées les unes avec les autres sans aucune attention; elles sont tenues ensemble avec des cordes ou des ficelles épaisses et cette collection est couverte de poussière et de toiles d'araignée ${ }^{42}$.

Cette description ne permet pas de savoir si les fragments étaient retournés dans un local attenant à la salle de prière ou conservés dans une autre partie du bâtiment. Dans le plan de la Grande Mosquée publié avec son étude de cette dernière en 1899, Henri Saladin indique que la petite pièce $\mathrm{J}$, située derrière la maqșūra à la droite du mihrab, « est la bibliothèque »; l'auteur commente un peu plus loin que « dans $\mathrm{J}$ il y a des armoires qui contiennent encore de beaux manuscrits ${ }^{43} »$. Mais la pièce L, opposée à $\mathrm{J}$, serait l'endroit où les fragments étaient conservés :

En L, ajoute Saladin, il y a des fragments de couvertures en bois... et des boîtes contenant des fragments de reliures et des restes de manuscrits 44 .

Dans leur livre consacré à l'étude des reliures de Kairouan, Georges Marçais et Louis Poinssot sont d'avis que les reliures découvertes dans un hall de la cour et à l'est du minaret ont été transférées à cet endroit parce qu'elles hébergeaient des parasites qui auraient pu endommager les livres qui subsistaient ${ }^{45}$. Ils avaient d'abord été stockés dans les armoires ou dans les caisses de la salle indiquée par Saladin, laquelle est devenue par la suite la bibliothèque selon Marçais et Poinssot.

En tout état de cause, après la publication de l'article de Muhammad Bayrām Bey, la collection fut traitée et un premier inventaire manuscrit fut achevé en 1901. Un inventaire récent est l'œuvre de Muḥammad al-Bahlī al-Nayyāl et d'Uthmān Jarad ; le premier auteur a également publié un petit livre très utile sur la bibliothèque et son histoire $^{46}$. À un moment donné, quelques pièces (reliures et feuilles) ont été transférées au musée du Bardo à Tunis pour être restaurées et éventuellement

41. «Essai sur l'écriture maghrébine», Nouveaux mélanges orientaux, Paris, E. Leroux, 1886, p. 85-112.

42. Muhammad BAyrām Bey, « Madīnat al-Qayrawān », Al-Muqtataf, vol. 21, 1897, p. 243.

43. H. Saladin, La Mosquée de Sidi Okba à Kairouan, Paris, E. Leroux, 1899, p. 37.

44. Ibid.

45. G. MARÇAIS et L. POINSSOT, Objets kairouanais. IX ${ }^{e}$ au XIII ${ }^{e}$ siècle. Reliures, verreries, cuivres et bronzes, bijoux, fasc. 1, Tunis/Paris, Tournier/Vuibert, 1948.

46. M. AL-NAYYĀL, al-Maktabat at-athariyya bi-l-Qayrawān. Arḍ wa-dalīl, Tunis, s.n., 1963. 
exposées dans des vitrines. Dans les années 1960, l'ensemble fut transféré à la Bibliothèque nationale de Tunis, à l'exception de quelques feuillets. En 1983, la collection retrouva son unité dans le musée d'Art islamique de Raqqāda, près de Kairouan - sauf les feuillets qu'on laissa au musée du Bardo. Un travail de restauration complet a été commencé en 1985-1986 avec le soutien de l'Allemagne.

La situation de la collection de Kairouan est paradoxale : alors que cette collection comprend des copies du Coran qui ont atteint une grande notoriété, comme le « coran bleu » ou le « coran de la Nourrice 47 », notamment à la suite d'expositions, et que la restauration a permis de remembrer en grande partie les manuscrits, elle ne dispose pas d'un catalogue. Pourtant, il s'agit d'une nécessité pour parer aux risques de vol : le mal est déjà fait puisqu'un nombre substantiel de feuillets ont été dispersés sans qu'il soit possible de s'assurer qu'il s'agit d'objets qui ont fait partie de la collection.

Pour terminer cette exploration de l'histoire de ces collections très particulières qui ont permis la sauvegarde des plus anciens manuscrits du Coran, j'évoquerai la découverte de Sanaa. Au début des années 1970, alors que le Yémen sortait à peine (en 1970) d'une longue guerre civile qui avait prolongé l'isolement où se trouvait la partie septentrionale du pays sous la monarchie restée en place jusqu'en 1962, les premières entreprises de préservation du patrimoine culturel se mettaient en place. Dans ce cadre, une mission italienne avait été chargée de dresser un bilan de l'état de la Grande Mosquée de Sanaa, un édifice dont l'histoire remonte aux premiers siècles de l'islam. Alors que les travaux étaient en cours, on découvrit entre le toit et le plafond de la salle de prière un ensemble de vieux parchemins et papiers : l'architecte Paolo Costa, qui supervisait l'entreprise, a été immortalisé en train d'examiner ces restes de manuscrits ${ }^{48}$. La question qui se posait était celle de la conservation de l'ensemble. Le Yémen disposait en la personne du qadi al-'Aqwa d'un responsable des antiquités qui disposait d'une réelle vision de ce qui devait être entrepris. Les manuscrits furent recueillis et, même si un très petit nombre d'entre eux fut peut-être séparé du reste à ce moment (des feuillets dont on soupçonne une origine yéménite ont été vus sur le marché des antiquités), l'essentiel attendit que soit trouvé un pays acceptant de prendre en charge financièrement et techniquement la préservation de l'ensemble ${ }^{49}$. Ce fut finalement à une équipe allemande que fut confiée cette tâche.

L'histoire ancienne de la collection reste mal connue : selon une source yéménite, des feuillets avaient déjà été retrouvés avant 1972, ce qui pose la question d'une dispersion des feuillets localement, mais aussi au-delà des frontières du Yémen. Il existe, d'autre part, peu de documents accompagnant les manuscrits qui permettent de déterminer leur origine. Un des très rares actes de waqf préservés indique un lien avec Sanaa, mais il n'est pas sûr que cette observation puisse être étendue à tous les manuscrits. De fait, l'une des découvertes les plus spectaculaires, des feuillets d'une

47. Pour ce dernier, voir F. DÉROCHE, «Le prince et la nourrice », Journal of Qur'anic studies, vol. 19, n 3 (N. DE CASTILLA (dir.), Qur'anic Manuscripts in the Western Islamic World), 2017, p. 18-33. Pour le « coran bleu », voir A. GEORGE, « Calligraphy, colour and light in the Blue Qur'an », Journal of Qur'anic Studies, vol. 11, $\mathrm{n}^{\circ}$ 1, 2009, p. 75-125 et bibliographie.

48. P. Costa, « La moschea Grande di San'â' », Annali dell'Istituto universitario Orientale di Napoli, vol. 34, 1974, p. 487 et p. 505-506.

49. Mașāhif Șan'ā', Kuwait : Dar al-Athār al-Islamiyyah, 1985, p. 20-21 [arabe]. 
copie in folio avec deux enluminures exceptionnelles qui représentent deux bâtiments généralement interprétés comme des mosquées, a été attribuée à la Syrie par l'éditeur des décors, Hans Caspar von Bothmer ${ }^{50}$. Si l'on admet cette origine, une hypothèse qui repose sur des arguments assez convaincants, il reste à savoir si le manuscrit a été envoyé au Yémen vers le moment où il a été réalisé, c'est-à-dire au tout début du VIII $^{\mathrm{e}}$ siècle selon l'hypothèse de von Bothmer, ou s'il y est arrivé à une date postérieure, une problématique que l'on retrouve d'ailleurs pour les trois autres collections que j'ai évoquées.

La même remarque que celle que je faisais à propos de Kairouan peut être répétée ici : alors que les fragments ont été restaurés et remembrés, il n'existe pas encore de catalogue. Pourtant, deux manuscrits au moins ont atteint une grande notoriété ${ }^{51}$. La situation est d'autant plus préoccupante que les circonstances actuelles laissent craindre que la collection souffre du conflit en cours. De plus, un autre ensemble de fragments avait été découvert peu avant le début de ce dernier, mais la découverte n'avait pas pu être exploitée.

En guise de post-scriptum à ce survol de ces genizôt musulmanes, je signalerai le cas de la collection de l'Astan-i Quds de Mashhad dont on ne sait pas trop s'il doit être ou non inclus dans le petit groupe que je viens d'évoquer. Il existe, en effet, dans le sanctuaire, un groupe de manuscrits dont les plus anciens remontent sans doute à la première moitié du VIII ${ }^{\mathrm{e}}$ siècle. La question des conditions de leur préservation est posée. Cette institution conserve en effet, d'autre part, un certain nombre de copies attribuées à des figures de premier plan du chiisme, en d'autres termes 'Ali et sa descendance. Ces copies datent dans l'ensemble du IX ${ }^{\mathrm{e}}$ siècle - un matériel très bien représenté dans les autres dépôts. La question est de savoir si les uns et les autres, qui apparaissent mélangés dans le catalogue, ont traversé ensemble les siècles ou si les manuscrits dépourvus de pedigree étaient conservés dans les mêmes conditions que ceux que j'ai évoqués précédemment, alors que les autres relèvent des reliques dont il a été question plus haut. Il est vrai que les frontières étaient sans doute poreuses dans tous les cas, un feuillet ou un manuscrit pouvant très bien être retiré d'un de ces dépôts pour être promu au statut de relique vénérable. Il convient de souligner que, dans la majorité des cas, les manuscrits qui ont ce statut sont des copies du IX ${ }^{\mathrm{e}}$ siècle (et parfois même plus tardives), très rarement du VIII ${ }^{\mathrm{e}}$ siècle.

Au sein de l'énorme production manuscrite musulmane, les manuscrits anciens du Coran copiés entre le $\mathrm{VII}^{\mathrm{e}}$ et le $\mathrm{X}^{\mathrm{e}}$ siècle (voire début $\mathrm{XI}^{\mathrm{e}}$ siècle) représentent une exception. Outre qu'ils sont majoritairement copiés sur du parchemin et très souvent dans un format particulier, leurs conditions de survie ont été particulières. En effet, les dépôts dont il a été question longuement représentent une forme de préservation très particulière des manuscrits. Même si, dans ces ensembles, figurent également des copies de textes non coraniques, voire dans d'autres langues, ces dernières représentent une faible minorité - à l'exception de Kairouan. Les copies sur papier du Coran postérieures aux $\mathrm{X}^{\mathrm{e}} / \mathrm{XI}^{\mathrm{e}}$ siècles ont certainement été aussi retirées de la circulation lorsque leur état ne permettait plus de les utiliser normalement, mais des indications çà et là laissent penser qu'elles étaient alors vouées à d'autres usages : on

50. H.C. VON BOTHMER, «Architekturbilder im Koran. Eine Prachthandschrift der Umayyadenzeit aus dem Yemen », Pantheon, vol. 45, 1987, p. 4-20.

51. Il s'agit, d'une part, du palimpseste (Șan'ā', Dār al-Makhțūtāat, Inv. 01-27.1) et, de l'autre, du grand coran omeyyade étudié par H.C. von Bothmer (Inv. 20-33.1, voir note précédente). 
sait ainsi que leur transformation en cartonnage pour servir d'ais à d'autres copies du texte révélé ou à des recueils de hadith était admise. La majorité des manuscrits coraniques plus récents qui nous sont parvenus a toutefois été préservée dans des bibliothèques de manière parfaitement conventionnelle. Notons toutefois qu'il existe dans la distribution chronologique du matériel, à la lumière de ce que nous savons actuellement, une incohérence : en effet, les manuscrits coraniques des $\mathrm{XI}^{\mathrm{e}}$ et $\mathrm{XII}^{\mathrm{e}}$ siècles, sur papier, sont comparativement peu nombreux par rapport aux centaines de copies sur parchemin qui ont été conservés. Est-ce la conséquence d'une chute dans la production, de disparitions pour des causes naturelles, d'une pratique de recyclage intense ? C'est un point qui mérite d'être pris en considération.

La question de la mise en place de ces structures réservées aux copies anciennes du Coran est difficile. Peu de données sont disponibles ${ }^{52}$. Le cas le plus favorable est celui de Kairouan: un inventaire établi en 1294 permet de savoir que des manuscrits anciens du Coran qui furent par la suite amassés dans un dépôt étaient encore disponibles dans la salle de prière ${ }^{53}$. Tous ? Sans doute pas. Mais un bon nombre du moins. Parmi les fragments de Damas, l'un d'eux porte une note d'un lecteur datée du $\mathrm{XII}^{\mathrm{e}}$ siècle : on peut en déduire que cet exemplaire était encore accessible à cette époque. Pour Fusțāt et pour Sanaa, la situation est obscure. Mais, bien entendu, les entrées dans les différents dépôts ont pu se poursuivre sur une longue période, le cas le plus clair étant celui de Damas.

Ce n'est que très récemment que ces manuscrits ont commencé à être pris en considération, tant comme témoignage sur la culture des premiers siècles de l'islam que comme source de premier plan pour notre compréhension de l'histoire du texte coranique à cette époque, mais aussi pour l'histoire de sa transmission matérielle. Une connaissance plus précise de ces dépôts est donc nécessaire pour préciser le contexte historique de cette production et de sa survie.

\section{Colloque - LE CoRAN DANS L'HISTOIRE CULTURELLE ET INTELLECTUELLE DE FUSȚĀT ENTRE LE VII ${ }^{\mathrm{e}}$ ET LE Xe SIĖCLE}

Colloque organisé au Collège de France les 6 et 7 juin 2018.

- Bouderbala Sobhi (faculté SHS de Tunis) : «La mosquée de 'Amr à Fusțāt : foyer de culte et de culture dans l'Égypte pré-fatimide»;

- Stéfanie Franke (Corpus Coranicum, Berlin Brandenburg Academy of Sciences and Humanities): "The language of ornaments-illuminated Early Qur'ānic manuscripts from al-Fusțāt »;

52. Des textes juridiques consacrés à cette question existent, comme celui d'époque tardive qu'a publié J. SADAN ( Genizah and genizah-like practices in Islamic and Jewish traditions. Customs concerning the disposal of worn-out Sacred Books in the Middle Ages, according to an Ottoman source », Bibliotheca Orientalis, vol. 43, 1986, p. 37-58). Mais il faudrait sans doute une enquête plus systématique pour situer la date d'apparition de ces préoccupations.

53. I. Chabbouh, «Sijill qadīm li-maktaba Jāmi' al-Qayrawān », Revue de l'institut des manuscrits arabes, vol. 2, no 2, 1956, p. 339-372 et pl. 1-7. Voir également F. DÉROCHE, «A note on the mediaeval inventory of the manuscripts kept in the Great mosque of Kairouan », in R. KERR et T. MILO (dir.), Writings and Writing: Investigations in Islamic Text and Script: In Honour of Dr Januarius Justus Witkam, Cambridge, Archetype, 2013, p. 67-86. 
- Patricia Roger-Puyo (Centre Ernest Babelon, CNRS, Orléans) : «Au plus près d'un corpus de corans provenant de la collection d'Asselin de Cherville, synthèse des observations et des analyses »;

- François Déroche (Collège de France) : «"Et ils voyagèrent...." »;

- Bassam Tahhan (chercheur indépendant) : «Le statut du Coran dans la Risāla de Shāfi '̄ì»;

- Walid Saleh (University of Toronto) : «The Heavenly Tablet and the Written Qur'ān: A new understanding of Early Mașāhifif»;

- Michael Marx (Corpus Coranicum, Berlin Brandenburg Academy of Sciences and Humanities) : «La relation entre la transmission écrite et la tradition savante : le cas des noms propres dans le Coran»;

- Michael Marx et Oliver Pohl (Corpus Coranicum, Berlin Brandenburg Academy of Sciences and Humanities) : «Paléocoran : quelques paramètres techniques »;

- Éléonore Cellard (Collège de France) : «Codex Amrensis I. Un facsimilé de Documenta Coranica» ;

- Mathieu Tillier (université Paris-Sorbonne) et Naïm Vantieghem (IRHT/CNRS) :

« Un Coran égyptien sur papyrus au tournant du VIII ${ }^{\mathrm{e}}$ siècle »;

- Catherine Louis (IRHT/CNRS), «II. Le palimpseste et la copie du Coran», Éléonore Cellard (Collège de France) : « Du copte à l'arabe : un nouveau palimpseste pour l'histoire du Coran au VIII ${ }^{\mathrm{e}}$ siècle. I. Réflexions sur la genèse du palimpseste »; - Hassan Chahdi (Collège de France) : «Les systèmes traditionnels de vocalisation du Coran à l'épreuve des données empiriques des manuscrits de Fusțāt » ;

- Morteza Karimi Nia (Encyclopaedia Islamica Foundation, Téhéran) : «From Fusțât to Iran: A survey of some old Kufic Qur'ans with possibly origin from Egypt »;

- Salome Beridze (University of Hamburg, CSMC) : «Textual variations and spelling in the manuscript Arabe 335 and affiliated fragments (Küfi B) »;

- Tobias J. Jocham (Corpus Coranicum, Berlin Brandenburg Academy of Sciences and Humanities) : "Variants readings in CBL 16151, a multi-layered manuscript form the $7^{\text {th }}$ century »;

- Edin Mahmutovic (Collège de France) : «Variant readings in the manuscripts of the Quranic library of Fusțāt. Examples ».

\section{RECHERCHE}

Dans le cadre du projet ANR franco-allemand Paléocoran, les recherches se sont poursuivies dans deux directions : d'un côté, l'histoire et le contexte de la Bibliotheca coranica de Fusțāt, de l'autre, des travaux spécifiques sur certains manuscrits du Coran appartenant à cet ensemble et sélectionnés en fonction de leurs particularités paléographiques. E. Mahmutovic, ATER au Collège de France, a travaillé à l'identification des systèmes de lecture tels qu'ils apparaissent sur des manuscrits anciens et a exposé les résultats de ses recherches lors du colloque organisé les 6 et 7 juin 2018. À cette occasion, H. Chahdi, post-doctorant du projet, a donné également les résultats de ses recherches sur les systèmes de vocalisation de manuscrits de Fusțât, tandis que E. Cellard, post-doctorante du projet, a présenté une découverte majeure qu'elle a faite d'un palimpseste où un texte coranique recouvrait une strate antérieure effacée en copte. De son côté, F. Déroche a évoqué les déplacements des 
manuscrits et les problèmes que cela pose pour l'histoire du Coran - sur laquelle il prépare un livre.

Le projet SICLE, senior grant de l'ERC, a été marqué par la tenue d'un colloque international en septembre 2017 : «Les bibliothèques à l'âge du manuscrit ». La rencontre a permis de confronter certaines des hypothèses que nous avons formulées sur la bibliothèque saadienne avec des résultats qui ont été obtenus par des spécialistes travaillant sur d'autres aires culturelles. Nuria de Castilla a parlé à cette occasion des manuscrits de poésie de la collection de l'Escorial pris de manière holistique. Les recherches sur la vie intellectuelle et culturelle du Maroc saadien se sont poursuivies ; une mission à Fès a notamment débouché sur la découverte de nombreux documents qui enrichissent notre connaissance de la période.

\section{PUBLICATIONS}

Cellard E., Codex Amrensis I, Leyde/Boston, Brill, coll. «Documenta coranica », vol. 1, 2018.

CHAHDI H., « Le paradoxe de la transmission des qirāāt : entre riwāya et qiyās ? », Journal of Qur'anic Studies, vol. 19, no 3, 2017, p. 103-133, DOI : 10.3366/jqs.2017.0304.

CHAHDi H. et Roger-PuYo P., «Étude d'un mușhaf maghrébin atypique du XVII ${ }^{\mathrm{e}}$ siècle : analyse conjointe des qirā'āt et des encres », Journal of Qur'anic Studies, vol. 19, n 3, 2017, p. 144-163, DOI : 10.3366/jqs.2017.0306.

DÉROCHE F., « Codicology », Encyclopaedia of Islam (3e éd.), 2017, DOI : 10.1163/15733912_ei3_COM_24409; en ligne : https://referenceworks.brillonline.com/entries/ encyclopaedia-of-islam-3/codicology-COM_24409?s.num=0.

DÉroche F., «Histoire du Coran : Texte et transmission », Annuaire du Collège de France 2015-2016. Résumé des cours et travaux, $\mathrm{n}^{00} 116,2018$, p. 281-292, DOI : 10.4000/annuairecdf. 12889 .

DÉroche F., «Le prince et la nourrice », Journal of Qur'anic Studies, vol. 19, n 3, 2017, p. 18-33, DOI : $10.3366 /$ jqs.2017.0300.

DÉROCHE F., «Lettrés et artistes chrétiens au temps des Omeyyades », in G. RAHAL et H.-O. LuTHE (dir.), Promissa nec aspera curans. Mélanges offerts à Marie-Thérèse Urvoy, Toulouse, Les Presses universitaires/Institut catholique de Toulouse, 2017, p. 263-273.

DÉroche F. et ZINK M. (dir.), Patrimoine et musées de l'Afrique du Nord, Paris, Éditions de Boccard, 2018.

DÉroche F., Le Coran, Paris, PUF, coll. «Que sais-je ? », vol. 1245, 2017, 5 e édition, http:// www.cairn.info/le-coran--9782130787266.htm.

DÉroche F., «A Qur'anic script from Umayyad times: Around the Codex of Fustat », in A. George et A. Marsham (dir.), Power, Patronage and Memory in Early Islam. Perspectives on Umayyad Elites, New York, Oxford University Press, 2018, p. 69-80.

Martínez de Castilla N., « A Bilingual 'Morisco Qur'an' with Thirteen Lines to the Page », Journal of Qur'anic Studies, vol. 19, n 3, 2017, p. 34-44, DOI : 10.3366/jqs.2017.0301. 
